\title{
Benchmarking set for domestic smart grid management
}

\author{
M.G.C. Bosman, V. Bakker, A. Molderink, J.L. Hurink, G.J.M. Smit
}

\begin{abstract}
In this paper we propose a benchmark for domestic smart grid management. It consists of an in-depth description of a domestic smart grid, in which local energy consumers, producers and buffers can be controlled. First, from this description a general benchmark framework is derived, which can be used as a guideline to create benchmark sets to compare domestic smart grid management methodologies. Secondly, an implementation of such a benchmark set is discussed in full detail, to give an example on how to use the framework to create a benchmark set. The application area and validity of a benchmark set can be clearly defined and checked, by using the general framework.
\end{abstract}

Index Terms-benchmark, microgeneration, domestic smart grid.

\section{INTRODUCTION}

In the last decades, more and more stress is put on the electricity supply and infrastructure. On the one hand, electricity usage increased significantly and became very fluctuating. Since the maximum peak usage defines the generation and grid capacity, the required capacity has increased. Furthermore, due to the fluctuations in usage (and therefore in required generation) the generation efficiency decreased [1].

On the other hand, reduction in the $\mathrm{CO}_{2}$ emissions and introduction of generation based on renewable sources are important topics today. However, these renewable resources are mainly given by very fluctuating and uncontrollable sun-, water- and wind-power. For this reason, supplemental production is required to keep the demand and supply in balance, resulting in an even more fluctuating generation pattern for the conventional power plants. Finally, the introduction of new, energy efficient technologies such as electrical cars can result in an even further fluctuating electricity demand. If electrical cars are charged in an uncontrolled way, this may result in high peak demands of electricity since these vehicles often will be charged in the evening and need to be charged fast to ensure enough capacity for the upcoming trip. Lowering the peaks in demand is desirable to improve the usage of the available grid capacity.

A solution for these problems may be to transform domestic customers from static consumers into active players in the production process. More and more new technologies with controllable load and generation are developed, such as controllable white goods and micro-generation. Furthermore, domestic energy storage of both heat and electricity is becoming quite common. At the moment, multiple projects are working

M.G.C. Bosman, V. Bakker, A. Molderink, J.L. Hurink and G.J.M. Smit are with the Department of Electrical Engineering, Mathematics and Computer Science, University of Twente, P.O. Box 217, 7500 AE, Enschede, The Netherlands, contact: m.g.c.bosman@utwente.nl. on control methodologies to optimize the consumption profile of individual households (among others [2], [3], [4]). The goal of these methodologies is to use the optimization potential to 1) optimize efficiency of current power plants, 2) support the introduction of a large penetration level of renewable sources (and thereby facilitate the means that are needed for $\mathrm{CO}_{2}$ reduction) and 3) optimize usage of the current grid capacity.

Comparing the effect, the strengths and the weaknesses of the different methodologies is very hard since different scenarios and use cases are used to verify them. Details of the tests are often not given so it is not possible to use the tests for another methodology. In this paper we present a benchmark framework that can be used to compare the different methodologies. To our knowledge the smart grid literature lacks such a set that can be used to compare several developed energy management initiatives. This benchmark is especially designed for the domestic energy infrastructure. Within the benchmark framework, test sets can be defined. We defined such an initial (benchmark) test set; the data required for this set is available on our website. However, we encourage others to extend the framework with more test sets, scenarios and use cases.

\section{A. Benchmarking vs. methodology development}

Note that this benchmark framework is designed to test domestic energy management methodologies at different criteria. An example is the ability to create stability under fixed and varying global conditions, while maximizing the energy efficiency in this smart grid. Although we try to set up realistic scenarios, the current benchmark set has a tendency to focus on the more extreme cases, in which real action is required to generate results. The different instances of this benchmark framework are not intended to be solved separately to optimality using an approach that is specifically designed for each test set. If another test set is created within the benchmark framework, the same generic methodology should be used to compare the quality of this methodology at varying (hard) circumstances from the different test sets.

The rest of this paper is structured as follows. Section II displays the situational context of a domestic smart grid. Also, the benchmark framework is derived here. Section III gives the requirements that our implementation of a benchmark set should fullfill. In sections IV and V this first benchmark set is defined in more detail. 


\section{SituATIONAL PERSPECTIVES: FROM DOMESTIC SMART GRID TO BENCHMARK FRAMEWORK}

In this section we define the context of our notion of a domestic smart grid and the implications from this context on the benchmark which is developed. In general, a smart grid is a network of energy-related devices (e.g. energy-producing, -consuming and -storing devices in buildings, power plants, wind mills) that are interconnected and can communicate, in which certain stakeholders (e.g. grid operators, building owners, electricity suppliers) can manage these devices to work towards their objectives (e.g. grid stability, energy efficiency optimization, $\mathrm{CO}_{2}$ reduction, profit maximization). In a domestic smart grid the focus is on the actions that owners of (small) buildings can take locally in order to optimize their (mutual) objective(s) (energy management). These actions are most often automized with a controlling algorithm to relieve the residents. Especially, energy management often means controlling of energy flows in buildings to optimize the electricity import/export profile.

\section{A. Benchmark framework}

Basic part of the benchmark framework of a domestic smart grid is the definition of the buildings: how many buildings are present, what is the energy demand of every building, what devices are installed, etc. Based on this the amount of schedulingsfreedom and therefore optimization potential can be deducted. Next, parameters of the control need to be defined: on which level can decisions be taken (within the house or on a global level), can predictions be used, what information can be exchanged, etc. Furthermore, the objective needs to be defined and a measure how to criticize the results. So, the concept of a domestic smart grid can be narrowed down to a description of:

1) buildings - the parameters and (energy) requirements of the buildings that are involved in this grid;

2) control - the parameters of the control algorithms (level, with/without predictions, etc.);

3) objective - the optimization objective;

4) measure - the measures to criticize the results.

These four elements are the building blocks for the benchmark framework. In a benchmark (test) set, which can be seen as an implementation of the framework, of course a few configuration parameters like the time granularity (time interval length of the simulation) need to be defined too.

\section{BENCHMARK SET}

An instance of the benchmark framework is defined by a description for each of the above mentioned building blocks and some additional parameters. In this paper a number of descriptions are given for each building block to be able to define a set of tests and construct a benchmark set. However, it is only a subset of possible scenarios and objectives, so we encourage others to extend this set and add descriptions to it, and to create new sets within the given framework. The goal of this paper is to setup a generic benchmark framework to compare smart grid management solutions; we give a number of descriptions to explain the setup of the benchmark framework and to create a first benchmark. All data used for the current benchmark is available on our website ${ }^{1}$, extensions will also become available on the website.

\section{A. General description of the set}

The parameters and requirements of the buildings are based on a model of a single house. This model is based on a generic framework consisting of (interconnected) models of the different parts of a house. The framework is described below, the next section gives a number of (simplified) models for the different parts. Based on the models and available appliances in every house the actions that can be taken to work towards the given objective can be deducted. Furthermore, a description of the objective is required as well as a description of how to measure upto which degree the objective is reached. These elements need to be addressed by the benchmark set to be able to define scenarios properly.

The scope of the benchmark set is (in first instance) on a neighbourhood level, the number of houses behind one single transformer. So, the number of houses in a test is 100-200. The houses are splitup in groups of 25 similar houses (number of residents, size of the house, insulation, etc.) since combining 25 houses into one set levels out demand peaks caused by starting appliances (which is realistic) but still a mix of housetypes can be used for a neighbourhood.

The single house framework models a house on the device level, since the behavior of individual devices is optimized. Houses contain multiple devices and exchange energy with the environment (e.g. gas import, electricity import/export). An example of a model of a house is shown in Figure 1.

Multiple types of energy can flow through the house (e.g. gas, electricity, heat). These types of energy are converted, buffered and consumed by devices, so we identify converting, buffering and consuming devices. Furthermore, energy can be exchanged with the environment. A detailed description of the underlying model we use can be found in [5].

We require easily reproducible and realistic parameter settings. To get realistic parameters, randomization is used for some parameters. However, randomization is hard to reproduce, so the semi-random decimal development of the number $\pi$ is used for randomization.

\section{DETAILS OF THE BENCHMARK SET}

This section gives a first implementation of the basic elements of the building blocks defined earlier. We propose an initial domestic grid, focusing on a description of the buildings, the way to control them, and the objectives and ways to measure the quality of the used control method.

\section{A. Buildings}

The benchmark set uses 100 houses in each scenario. A group size of 100 is of the same order of magnitude as a neighbourhood in a city. Also, we assume that this is sufficiently

\footnotetext{
${ }^{1}$ http://et.ewi.utwente.nl/
} 


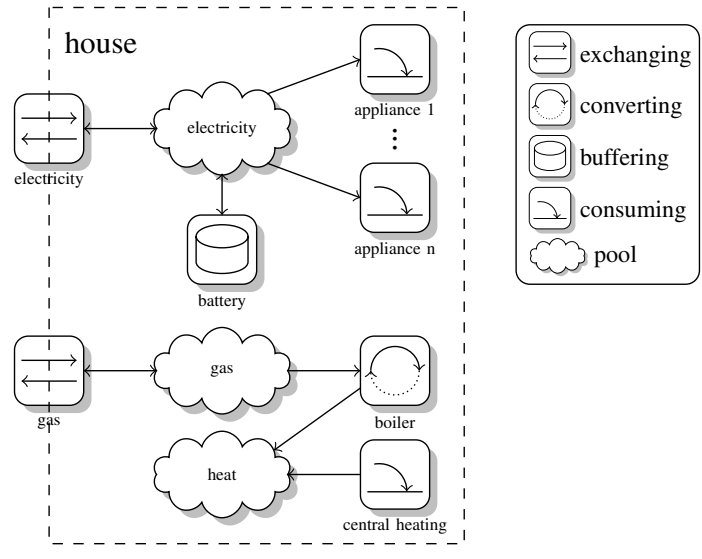

Fig. 1. Model of the house

large to distinguish between the individual aspects of the houses and the total effect of the group on the grid. The size is not changed among different benchmark instances, since we expect to have overwon small size scalability problems in the sense of our measures and objectives.

As described above, each benchmark uses a set of houses. Within each house, different kinds of appliances are available which determine the energy demand of the household. Goal of the methodology is to control all the appliances within a building, resulting in a certain energy profile. As described in the model, multiple energy-types can be used, but for now only electricity and heat are described. Consequentially we assume the required other resources like natural gas to be available abundantly, i.e. with no restrictions on the supply.

Appliances can be categorized, which will be described in the following sub sections.

1) Consumers: A building consists of consuming appliances. Below we address heat and electricity related parameters for these appliances.

Heat - This heat demand for the buildings is given for two days, a winter and a summer day. These two days are chosen such that the extreme cases can be analyzed. In general, we assume that the heat demand consists of central heating and hot tap water demand. In summer, no/little central heating is required and the heat demand is dominated by the tap water demand. During winter, central heat demand is added to the basic hot top water demand. As a consequence, the heat profile of a house has two peaks ${ }^{2}$.

The heat demand is expected to be higher during the day than during night. Therefore, different factors are used while generating the central heating demand during the working day. For the hot tap water demand, two peaks are expected.

To represent the heat demand sets of heat vectors $S^{s}=$ $\left\{\right.$ heat $_{1}^{s}, \ldots$, heat $\left._{100}^{s}\right\}$, are created, with each vector heat ${ }_{i}^{s}$ of size 24. If in some scenario a higher resolution of the time intervals is used, we simply downscale these hourly values (no interpolation between hours is done). The hourly vectors are generated as in Algorithm 1, with $w=4$ and

${ }^{2}$ Derived from gas usage patterns in The Netherlands $s$ varying from 0 to 3 (in the winter case) and from 20000 to 20003 (in the summer case). In the winter case the daily heat demand is assumed to be $54 \mathrm{kWh}$; in the summer case $13 \mathrm{kWh}^{2}$. In winter, this equals the average total heat demand, if the hourly demand (in Wh) is picked uniformly from the interval $I_{\text {winter }}=[500,4000]$. In the summer cases, the interval $I_{\text {summer }}=[300,783]$ is used. The function create_heat $\left(p, I_{\text {season }}\right)$ creates a heat demand based on the random number $p$ and the interval $I_{\text {season }}$ (using a uniform distribution over the interval $I_{\text {season }}$ ). Using the decimal development of $\pi$ in samples of four digits length , semi-random numbers are used to produce 24 hourly heat demands. These 24 values are then ordened, using 24 semi-random numbers as probabilities, to form a pattern with two peaks. First, two hours are selected from the corresponding sets $\{7,8,9,10\}$ and $\{18,19,20,21\}$ and the two highest of the 24 values are assigned to these selected hours. The function $\operatorname{match}(p, F)$ selects the hour from the set $F$, where the probability $p$ is used to pick the hour in the set $F$ following a uniform distribution. Then, the remaining values $T_{\text {unassigned }}^{i}$ are, in decreasing order, assigned to an hour, where this hour can only be chosen from the set of unassigned hours that differs one from an already assigned hour (i.e. the set freeplaces that is created by the function freeplaces $\left(\right.$ heat $\left._{i}^{s}\right)$, which assigns immediate neighbouring hours of already assigned hours to a set). The semi-random numbers and the function match $(p, F)$ are used again to decide which of these hours in freeplaces is assigned the given value.

So far only real heat demand is characterized. In some cases (see Section IV-B1) also a heat demand prediction is required. Since we want the prediction to characterize the general behaviour of the heat demand, but simultaneously to differ enough from the real demand to create a 'prediction effect', we create the prediction vectors heatpred ${ }_{i}$ as follows. The maximum deviation for the heat demand of building $i$ to the average heat demand for this building for the four cases of $s$ (per season case) is calculated. The heat demand corresponding to this maximum deviation is rounded to the nearest multiple of one thousand and assigned to heatpred ${ }_{i}$.

Electricity - Some appliance have some scheduling freedom, while other appliances must be directly used by the residents. For example, a freezer or fridge has to maintain a certain temperature range and is autonomous in how to achieve this. On the other hand, television sets or a coffee machine have to be supplied when switched on. Therefore, we divide the total electricity demand into two parts: controllable and non-controllable demand.

For the non controllable part a general pattern is given, that is equal for all houses ${ }^{3}$. This pattern is given in Table I.

We consider fridges, freezers, washing machines and dryers as controllable consumers. We assume that once a washing machines or dryer is switched in, the methodology is allowed to start the work to be done on a later time stamp.

Temperature control of fridges and freezers is usually done by a simple thermostat, which results in a more or less regular electricity consuming pattern. However, we allow

${ }^{3}$ Based on measurements of 14 electrical appliances 


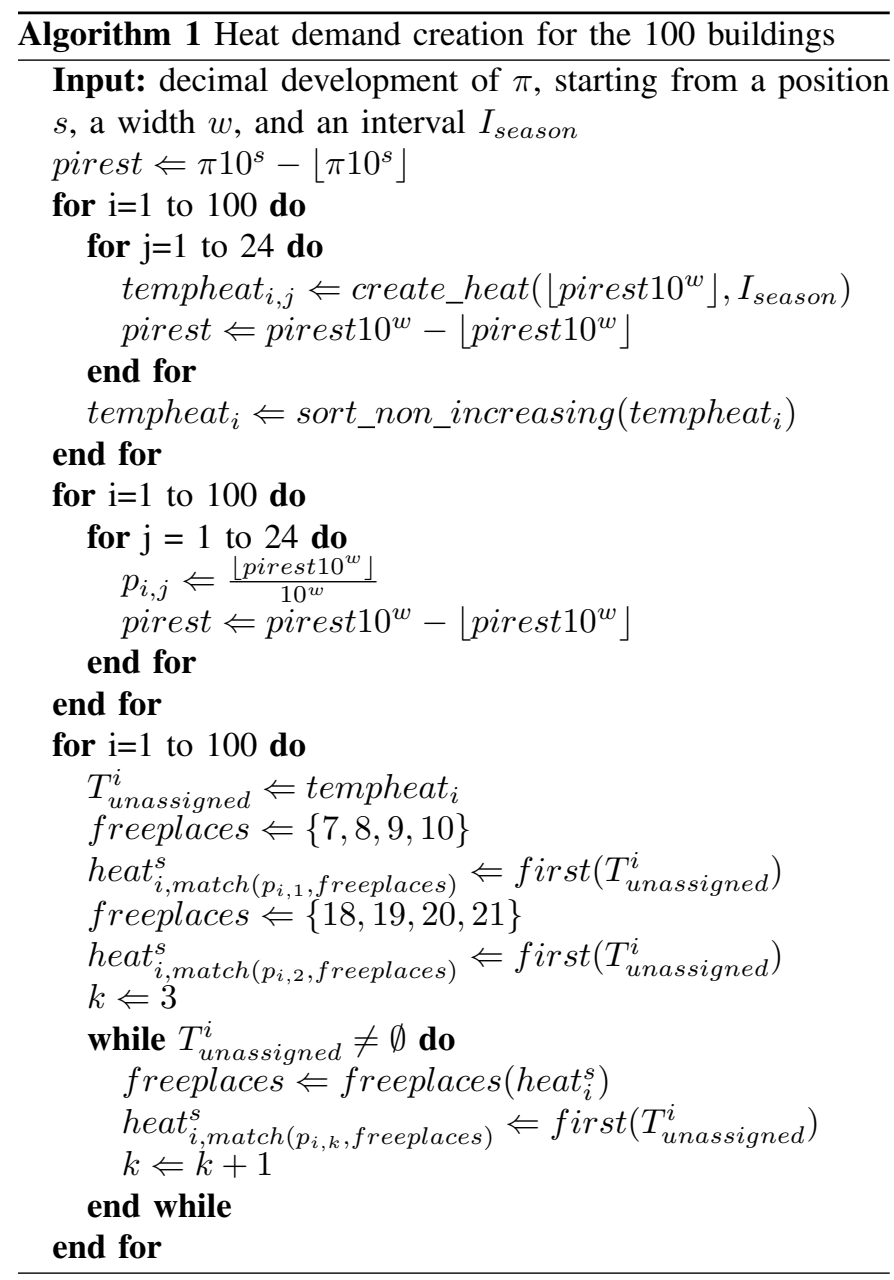

TABLE I

NON CONTROLLABLE ELECTRICITY DEMAND (IN INTERVALS OF 5 MINUTES LENGTH)

\begin{tabular}{cc|cc|cc} 
interval & demand $(\mathrm{W})$ & interval & demand $(\mathrm{W})$ & interval & demand $(\mathrm{W})$ \\
\hline $1-84$ & 95 & 181 & 2338 & 235 & 288 \\
85 & 1055 & $182-186$ & 238 & 236 & 2738 \\
$86-96$ & 95 & $187-191$ & 358 & 237 & 288 \\
$97-101$ & 358 & $192-204$ & 238 & 238 & 2738 \\
$102-114$ & 238 & $205-209$ & 358 & 239 & 1458 \\
$115-119$ & 358 & $210-222$ & 238 & 240 & 2948 \\
$120-132$ & 238 & $223-227$ & 358 & $241-242$ & 448 \\
$133-137$ & 1608 & 228 & 238 & $243-249$ & 328 \\
138 & 1488 & 229 & 2888 & $250-255$ & 408 \\
$139-150$ & 238 & 230 & 2738 & $256-260$ & 528 \\
$151-155$ & 358 & 231 & 288 & 261 & 408 \\
$156-168$ & 238 & 232 & 2738 & $262-267$ & 358 \\
$169-173$ & 358 & 233 & 288 & $268-273$ & 175 \\
$174-180$ & 238 & 234 & 2738 & $274-288$ & 95
\end{tabular}

two extended options to this control. First, various levels of power consumption can be chosen. Each level has a different influence on the temperature decrease, as shown in Table II. Secondly, the cooling is allowed to start at each possible level within the given limits. So, both the level of power level can be chosen and the timing can be shifted. Initially, the temperature of a fridge is set to $5.5^{\circ} \mathrm{C}$ and the temperature of a freezer to $-22.5^{\circ} \mathrm{C}$.

The consumption patterns of a washing machine and a dryer are given in Table III. The washing machine needs to run once in the interval $[7 \mathrm{~h}, 18 \mathrm{~h}]$ and the dryer in the interval $[18 \mathrm{~h}, 24 \mathrm{~h}]$.
TABLE II

CONTROLLABLE FRIDGES AND FREEZERS

\begin{tabular}{cccccc} 
& & \multicolumn{2}{c}{$\Delta$ temp. $\left({ }^{\circ} \mathrm{C} / \mathrm{min}\right)$} & \multicolumn{2}{c}{ temp. limits $\left({ }^{\circ} \mathrm{C}\right)$} \\
appliance & demand $(\mathrm{W})$ & on & off & lower & upper \\
\hline fridge & 100 & -0.2 & +0.06 & 4 & 7 \\
freezer & 50 & -0.12 & +0.06 & 4 & 7 \\
& 200 & -0.6 & +0.15 & -27 & -18 \\
& 100 & -0.36 & +0.15 & -27 & -18
\end{tabular}

TABLE III

CONTROLLABLE WASHING MACHINES AND DRYERS (IN INTERVALS OF 5 MINUTES LENGTH)

\begin{tabular}{cccc|cc}
\multicolumn{2}{c}{ washing machine } & \multicolumn{4}{c}{ dryer } \\
interval & demand $(\mathrm{W})$ & interval & demand $(\mathrm{W})$ & interval & demand $(\mathrm{W})$ \\
\hline $1-2$ & 0 & $1-2$ & 0 & $15-16$ & 1570 \\
$3-4$ & 2270 & $3-4$ & 1570 & 17 & 150 \\
$5-6$ & 100 & 5 & 150 & $18-19$ & 1570 \\
7 & 400 & $6-7$ & 1570 & 20 & 150 \\
8 & 100 & 8 & 150 & $21-22$ & 1570 \\
9 & 400 & $9-10$ & 1570 & 23 & 150 \\
& & 11 & 150 & $24-25$ & 1570 \\
& & $12-13$ & 1570 & 26 & 150
\end{tabular}

2) Buffers: Regarding buffers, we also specify both heat and electricity buffers.

Heat - We emphasize that all heat demand has to be fulfilled by the methodology. To facilitate this hard requirement all houses are equipped with a heat buffer with capacity $H C=10 \mathrm{kWh}$, which initially contains $H B=5 \mathrm{kWh}$ of extractable heat. In our set, eventual heat loss of a buffer is already incorporated in the heat demand. Furthermore, we assume that in- and outflow of a heat buffer is unrestricted, i.e. all heat demand/production can be provided/absorbed by the heat buffer. To allow for variation, a second type of heat buffer is introduced with characteristics $H C=20 \mathrm{kWh}$ and $H B=10 \mathrm{kWh}$.

Electricity - As batteries we propose two types. The first type concerns normal sized car batteries that are installed in buildings and, thus, can be used continually during the time horizon. Second, we introduce batteries that are used in an electric car, that can only be used when the car is at home. Moreover we have specific requirements on the battery level at the end of the period in which the car is at home. For the normal sized batteries we use capacities of $480 \mathrm{Wh}$ and $960 \mathrm{Wh}$ and a maximum charge/discharge power of $96 \mathrm{~W}$. Although it is convenient to use other units, we specify the battery characteristics in $\mathrm{W}$ and $\mathrm{Wh}$ to comply to the setup of the benchmark set. We assume an efficiency of $90 \%$. The second type of battery is a $53 \mathrm{kWh}$ battery in an electric car. We limit the maximum charge/discharge power in the building to $16.8 \mathrm{~kW}^{4}$. The electric car is available between $18 \mathrm{~h}$ and $7 \mathrm{~h}$ the next day. It is only used in the benchmark set if horizons of more than one day are considered.

3) Converters: The heat can be produced by a conventional boiler, a microCHP (Combined Heat and Power) or a heat pump. The conventional boiler system has its maximum heat output set to $32 \mathrm{~kW}^{5}$, which leads to an average run time of 1.7 hour per day, if the start and finish heat level of the buffer are equal. The microCHP delivers $8 \mathrm{~kW}$ maximum heat output,

\footnotetext{
${ }^{4}$ http://www.teslamotors.com/

${ }^{5} \mathrm{http}: / /$ www.daalderop.nl/
} 
combined with a maximum electricity output of $1 \mathrm{~kW}^{6}$. To create diversity, also (future) microgenerators with a maximum electricity output of $2 \mathrm{~kW}$ and a maximum heat output of $7 \mathrm{~kW}$ are given, as well as microgenerators with a $4 \mathrm{~kW} / 5 \mathrm{~kW}$ output relation. A startup time of 12 minutes is assumed, in which both power outputs linearly increase from 0 to their respective maximum values. Also a shutdown period of 5 minutes is applied, in which a linear decrease to 0 takes place. Since during the startup period the maximum energy efficiency is not utilized, a minimum total runtime of half an hour is set, once a microCHP has been started. This minimum waiting time of half an hour is also applied after a microCHP has been stopped, to promote longer runs and prevent wearing. The heat pump is modeled to consume $1 \mathrm{~kW}$ of electricity at operation. It produces, after a linear increase of 10 minutes, a maximum heat output of $3 \mathrm{~kW}$. As alternatives, $4.5 \mathrm{~kW}$ and $6 \mathrm{~kW}$ outputs are used.

\section{B. Control}

In this benchmark set, we use discrete intervals of a given length, in which certain decisions must be made. Note that all decisions are fixed for the complete length of the interval; letting the microgenerator run for 1 minute and then shutting it down is not allowed when using intervals of 5 minutes length. This subsection addresses two elements that play a crucial role in the decision making process:

- the information that can be used in an interval;

- the actions that can be chosen from.

1) Information: Regarding information, we allow two cases of communication. In a privacy case buildings cannot use information of other buildings in decision making. In a full knowledge case full communication between buildings is allowed in the scenarios. This implies that the methodology has full knowledge about every local parameter in the domestic grid and can use the knowledge of a building in its decision making for another building. This knowledge is only constrained by the moments in time that (demand or global) data becomes available. In an offline case this information is available at the start of the scenario. This information can be processed before any decision is taken. In an online case information about the upcoming interval becomes available at the start of this interval. Fixed decisions for this interval should be made before new information about the next interval becomes available. In an online-prediction case the information becomes available in an online way, and an offline prediction of the demand can be used next to this information.

2) Decisions: It is important to define the actions that the decision maker can take in the given scenarios. We distinguish between three types of control:

1) microgeneration;

2) storage techniques;

3) demand side load management.

Regarding microgeneration, decisions can be made to have the generator on or off. Of course the methodology should

\footnotetext{
${ }^{6} \mathrm{http}: / / \mathrm{www}$.whispergen.com/
}
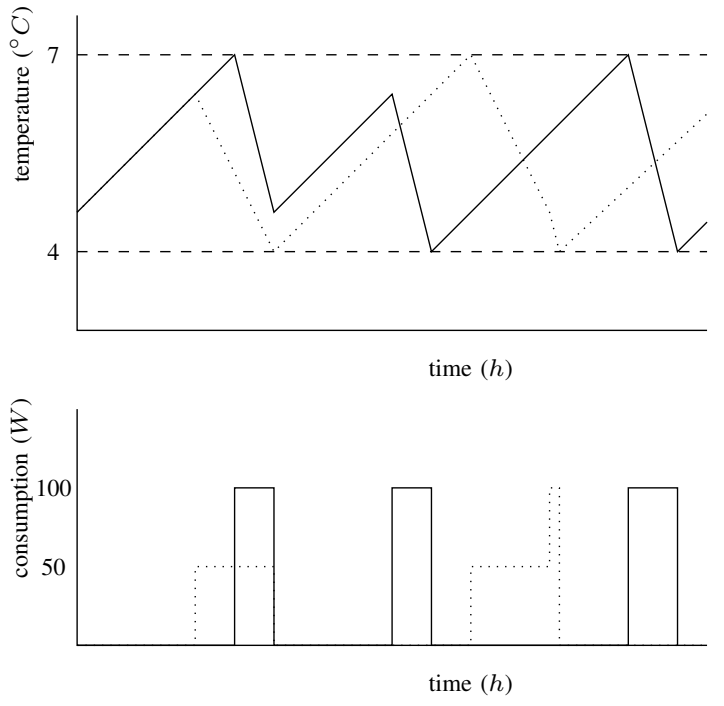

Fig. 2. Shifting possibilities for electricity consumption

take the minimum runtime and offtime constraints and the limitations of the heat buffer (it is not allowed to waste heat) into account. If in a certain interval no decision can be made due to a conflict between runtime/offtime constraints on the one hand and heat buffer constraints on the other, a choice must be made to prevent violating the runtime/offtime constraint. The heat shortage/surplus that arises is penalized for.

No heat related storage decisions must be made; the generated heat is stored in the heat buffer, immediately supplied to the heat demand or accounted for as (penalized) heat surplus/shortage. Concerning electricity storage, the battery can be used to shift electricity production/consumption to more profitable times, within the limitations of the battery (battery capacity and maximum charge/discharge). The electric car battery needs to be filled at the end of the available period.

The electricity demand side load management focuses on two kinds of appliances which are mentioned before. For fridges and freezers the power consumption can be chosen as pictured in Figure 2. Note that, during the cooling process, the choice for a certain consumption level can be adapted. However, during an interval this choice needs to be fixed. Of course the temperature limits need to be satisfied. For the washing machine and the dryer, in the offline case a set of intervals is given in which the washing machine should start and complete a run. In the online case, at a certain interval the interval is given before and/or in which the appliance should have finished a complete run. As long as a choice is postponed to start this run, new information becomes available which can be used in the decision process. However, in this case it cannot be decided to start the run in a previous interval. A washing machine and a dryer both have to run exactly once a day.

\section{Objectives}

We define two types of global objectives. The first type concerns peak shaving, a situation where the electricity import/export pattern of the group of buildings is controlled in 
such a way, that a flat line is approximated (the peaks in the import/export pattern are 'shaved'). This type is suited for use in an offline, online and online-prediction case, since the objective of peak shaving is to control the internal production/consumption. So the objective is not influenced from outside the domestic grid. Especially in the latter two cases, if we can predict or know our internal parameters in advance, we are able to produce a global objective and control the group of houses in an offline setting.

The second type deals with fluctuation control, a situation under uncertainty where we want to react on the fluctuating output of a small windmill park. This type is only suited for use in an online case. If we know the internal parameters in advance, we are still not able to know (and work towards) our objective.

1) Peak shaving: We define two peak shaving patterns. The goal of the first pattern is to flatten the total electricity output of the microgenerators. An average value peak $k_{\text {prod }}$ is calculated by taking the average electricity production of all buildings, when they would generate exactly the amount of heat that is needed to fullfill the real heat demand. We allow small deviations to above and below this average of $1 \mathrm{~kW}, 5$ $\mathrm{kW}$ and $10 \mathrm{~kW}$ in respective cases. Violation of these limits is penalized in the objective function. The goal of the second pattern is to flatten the pattern that includes both generated electricity and consumed electricity (including storage). An average value peak $k_{\text {prod } \mid \text { cons }}$ is calculated by taking the average electricity production minus consumption. Again, deviations of $1 \mathrm{~kW}, 5 \mathrm{~kW}$ or $10 \mathrm{~kW}$ are assigned to this profile. These values are chosen such, that it is hard to find a feasible solution within these bounds. In this way the main focus is on minimizing the deviation outside these bounds, resulting in an objective that can be measured.

2) Fluctuation control: For the windmill park situation we also define two objectives. The first is to produce a matching pattern with the total output of the microgenerators, while the second one is to produce a matching pattern with production, consumption and storage. The pattern that has to be matched is the following. The average values peak $k_{\text {prod }}$ and peak $k_{\text {prod|cons }}$ are the basic of this pattern. For each 1 minute interval a fluctuating vector $f=\left(f_{1}, \ldots, f_{1440}\right)$, where $f_{j} \in[-5 k W, 5 k W]$ is added to form the wind mill patterns fluc $_{\text {prod }}$ and $f l u c_{\text {prod } \mid \text { cons }}$ :

$$
\begin{array}{r}
f l u c_{\text {prod }}=\text { peak }_{\text {prod }}+f \\
f l u c_{\text {prod } \mid \text { cons }}=\text { peak } k_{\text {prod } \mid \text { cons }}+f
\end{array}
$$

The values $f_{j}$ are picked from a uniform distribution on the interval $[-5 \mathrm{~kW}, 5 \mathrm{~kW}]$ by using semi-random numbers of length 4 from the development of $\pi$ when starting from $s=$ 40000 .

\section{Measures}

As final step in the benchmark set measures need to be derived.

The criteria for the benchmark set consists of three parts. First, the feasibility of a solution to the benchmark instance' objective is checked and a measure is derived for the quality of infeasibility. Second, two measures for the quality of domestic smart grid control are derived. Finally, the computational effort needs to be specified.

For each part a score system is developed, which is calibrated to a certain lower limit and a certain upper limit. The score is scaled linearly, where the lower limit is set to 0 and the upper limit to 100 . In theory scores higher than 100 are possible, although this is not very likely.

1) Feasibility: In this benchmark set we propose locally a heat driven control and on top of that global, electricity driven limits given by the selected objective. Failure of the control methodology to subject to these constraints leads to certain penalty costs. These penalty costs lead to a sum of constraint violations (in $\mathrm{kWh}$ ); the higher this sum, the worse the violation. A sum of 0 means a feasible solution is found. This is the lower limit in the score $S_{o b j}$ of the score system for the objective. The upper limit is set to the sum of the generation or the sum of generation and consumption, depending on the objective. In this way, the maximum score is always below 100 .

2) Quality: Besides the objectives that are already incorporated in the benchmark via constraints (e.g. peak shaving) we focus on two measures of quality:

- profit;

- efficiency.

In profit maximization we try to maximize the average price per $\mathrm{kWh}$ produced electricity, if the generated electricity would have been sold on the electricity market. We define two sets of electricity prices in Table IV. The first one is a calm day at the electricity market; the second one is a highly fluctuating day.

TABLE IV

MARKET PRICES OF 01-09-2009 AND 29-10-2007

\begin{tabular}{cccc|cccc}
\multicolumn{9}{c}{ APX 01-09-2009 } & \multicolumn{5}{c}{ APX $29-10-2007$} \\
hour & $p_{j}(€ / \mathrm{MWh})$ & hour & $p_{j}(€ / \mathrm{MWh})$ & hour & $p_{j}(€ / \mathrm{MWh})$ & hour & $p_{j}(€ / \mathrm{MWh})$ \\
\hline 1 & 33.00 & 13 & 44.85 & 1 & 37.00 & 13 & 124.96 \\
2 & 26.50 & 14 & 49.93 & 2 & 29.65 & 14 & 135.00 \\
3 & 21.62 & 15 & 48.99 & 3 & 22.38 & 15 & 111.61 \\
4 & 19.84 & 16 & 44.93 & 4 & 19.01 & 16 & 103.96 \\
5 & 19.41 & 17 & 45.85 & 5 & 28.07 & 17 & 171.04 \\
6 & 20.73 & 18 & 45.57 & 6 & 37.04 & 18 & 500.00 \\
7 & 35.60 & 19 & 39.00 & 7 & 63.01 & 19 & 275.00 \\
8 & 41.10 & 20 & 30.11 & 8 & 91.06 & 20 & 187.57 \\
9 & 42.99 & 21 & 35.37 & 9 & 103.97 & 21 & 92.50 \\
10 & 44.93 & 22 & 37.00 & 10 & 179.89 & 22 & 66.50 \\
11 & 42.00 & 23 & 35.37 & 11 & 150.44 & 23 & 51.50 \\
12 & 54.93 & 24 & 34.50 & 12 & 242.80 & 24 & 47.00
\end{tabular}

The efficiency of the used appliances and imported electricity is pictured in Table V. This efficiency is expressed as the

TABLE V

ENERGY EFFICIENCY OF APPLIANCES AND IMPORT

\begin{tabular}{cc} 
appliance & efficiency $(\%)$ \\
\hline conventional boiler & 90 \\
microCHP & {$[0-90]$} \\
heat pump $(3 \mathrm{~kW})$ & {$[0-300]$} \\
heat pump $(4.5 \mathrm{~kW})$ & {$[0-450]$} \\
heat pump $(6 \mathrm{~kW})$ & {$[0-600]$} \\
electricity import (normal) & 50 \\
electricity import (peak) & 30
\end{tabular}

fraction of produced/consumed energy and the primary energy that is needed for this production/consumption. Note that the electricity that is used from the battery causes an additional energy loss of $10 \%$. 
As a measure for the quality of profit maximization and efficiency maximization a score system is developed. Scores $S_{\text {profit }}$ and $S_{\text {eff }}$ are assigned between 0 and 100. In profit maximization the lower limit is set to the smallest price of the day and the upper price to the highest price of the day. Regarding efficiency the lower limit is defined as an efficiency of $30 \%$ and an efficiency of $600 \%$ as the upper limit.

3) Computational effort: Finally the computational effort should be specified by mentioning the computation time and the system that is used. A score $S_{\text {comp }}$ is defined with, as calibration marks, 0 seconds as a lower limit and 1800 seconds as the upper limit. In case full communication is allowed between buildings, also mention the fraction of the computation time that is dedicated to this communication.

4) Total measure: Ultimately the goal of the control methodology is to minimize the objective violation, while maximizing both profit and efficiency, with as less computational effort as possible. Therefore we define the final score $S_{\text {final }}=w_{o b j} S_{o b j}+w_{\text {profit }}\left(100-S_{\text {profit }}\right)+w_{\text {eff }}(100-$ $\left.S_{\text {eff }}\right)+w_{\text {comp }} S_{\text {comp }}$. In our vision the four measures are given in decreasing order of importance, leading to weights $w_{o b j}=100, w_{\text {profit }}=10, w_{\text {eff }}=10$ and $w_{\text {comp }}=1$.

\section{SCENARIOS}

The current version of the benchmark set consists of the scenarios that are defined below. In designing these scenarios two aspects play an important role. First, the representation of a building and the mix of buildings that forms a neighbourhood needs attention. Next, the combinations that form a scenario need to be considered.

\section{A. Building setups and mixtures of buildings}

An important part of the modelling of the benchmark set is how a building is expressed. A building is characterized by its energy demand, microgenerator, appliances and buffers. In Table VI one possible combination of these characteristic parameters is given. Note that in each row exactly one parameter must be chosen, except for the controllable appliances which have to be picked all in each instance. Note that, in this basic version of the benchmark, the controllable and non controllable appliances are always available. However, in case demand side load management is not allowed, the controllable appliances must be controlled by a thermostate-like approach: only switching on when the upper temperature limit is reached, and only switching off when the lower limit is reached. The mix of buildings now is created by combining different building setups in portions of 25 similar houses. The total amount of buildings is 100; so, 4 (possibly different) sets of houses are combined to form the background for the domestic smart grid benchmark. In this benchmark we focus on heat led control; therefore we apply all possible combinations of elements for a fixed heat demand and calculate the average score for this set. Thus, if we speak of a building case with a certain heat demand in the next section, we mean the average of all building setups with this specific heat demand.
TABLE VI

PARAMETER CHOICE FOR A BUILDING CONFIGURATION

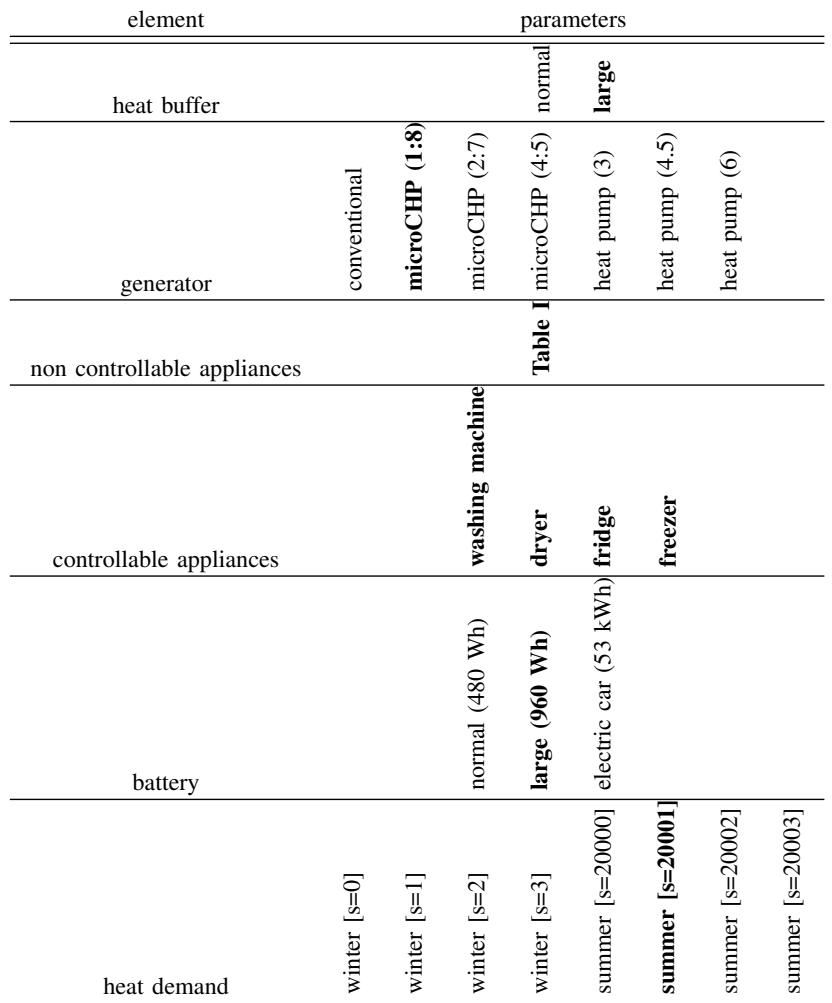

\section{B. Scenario types}

The benchmark framework and our benchmark set are divided into four building blocks. Since the actual cases need additional information, we loosen this 4-tuple description and use a more convenient one. A scenario is defined as a 5-tuple (building case (specific heat demand)|time granularity|control|objective|price). The control is specified by a 3-tuple (time control level|decision level|information availability). As basic instances of this benchmark set we propose some of the possible combinations that can be made from this definition of a scenario. We define three types of scenarios:

- peak shaving; where both electricity patterns peak $k_{\text {prod }}$ and peak $k_{\text {prod|cons }}$ should be matched offline, online or online-prediction by the sets of houses with all combinations of the four heat demands and other parameters, except for the electric car. We use full knowledge of the buildings. For all possible combinations of the above we want to test the quality of the control methodology against:

1) varying decision levels (i.e. using only local microgeneration, using local microgeneration and storage techniques, using demand side load management or using a combination of local microgeneration, demand side load management and storage techniques);

2) varying time granularity;

3) varying prices.

- wind fluctuation control; where we only compare online 
control on the $f l u c_{\text {prod }}$ and $f l u c_{\text {prod } \mid \text { cons }}$ patterns, under the same conditions as in the peakshaving scnearios. Again we test the quality in 3 dimensions:

4) decision levels;

5) time granularity;

6) price.

- electrical car; the introduction of large electricity draining appliances/ buffer providing appliances in the network is tested for a peak $k_{\text {prod }}$ objective, in an offline setting, using full knowledge about the neighbourhood. Can the control method 'peak shave' a week agenda (the car has to be used during daytime) by the use of local microgeneration, demand side load management and (other) storage techniques, where $50 \%$ of the houses has an electrical car? The following dimensions are considered:

7) time granularity;

8) price.

\section{CONCLUDING REMARKS}

In this paper a framework and a first approach towards a domestic smart grid benchmark is given. Extensions to the existing set or new sets are more than welcome. We can think of domestic wind mills, PV cells, biogas installations and more, each of which can be defined in a separate benchmark set, leaving the results of preliminary work unharmed.

The current benchmark is certainly not complete. However, to our knowledge it is the first set, defined in more detail, which can be used to compare different domestic smart grid control initiatives.

\section{ACKNOWLEDGEMENTS}

This research is conducted within the SFEER project (07937) supported by STW, Essent and GasTerra.

\section{REFERENCES}

[1] A. de Jong, E.-J. Bakker, J. Dam, and H. van Wolferen, "Technisch energie- en CO2-besparingspotentieel in Nederland (2010-2030)," Platform Nieuw Gas, p. 45, Juli 2006.

[2] A. Molderink, V. Bakker, M. Bosman, J. Hurink, and G. Smit, "A threestep methodology to improve domestic energy efficiency," in IEEE PES Conference on Innovative Smart Grid Technologies, 2010.

[3] J. Kok, C. Warmer, and I. Kamphuis, "Powermatcher: Multiagent control in the electricity infrastructure," in 4th international joint conference on Autonomous agents and multiagent systems. ACM, Jul 2005, pp. 75-82.

[4] D. Chassin and L. Kiesling, "Decentralized coordination through digital technology, dynamic pricing, and customer-driven control: The gridwise testbed demonstration project," The Electricity Journal, vol. 21, pp. 5159, 2008.

[5] V. Bakker, A. Molderink, M. G. C. Bosman, J. L. Hurink, and G. J. M. Smit, "On simulating the effect on the energy efficiency of smart grid technologies," in Proceedings of the 2010 Winter Simulation Conference submitted, 2010.

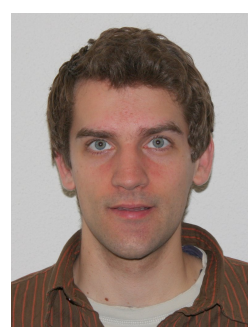

Maurice G.C. Bosman received his M.Sc. degree in Applied Mathematics from the University of Twente in February 2008. Currently he is a PhD student in the CAES and DMMP groups at the faculty of Electrical Engineering, Mathematics and Computer Science at the University of Twente. His research interests include energy efficiency, scheduling and online algorithms.

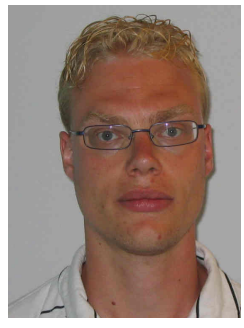

Albert Molderink was born in Heerenveen (The Netherlands) in 1983. He received his B.Sc and M.Sc. degree in Computer Science from the University of Twente, Enschede, The Netherlands, in respectively 2004 and 2007. In addition, he received a Electrical Engineering minor certificate. His research focus is on algorithms to optimize energy streams within a house. His research interests include energy efficiency, mathematical modelling and optimizations, algorithm development and embedded hardware.

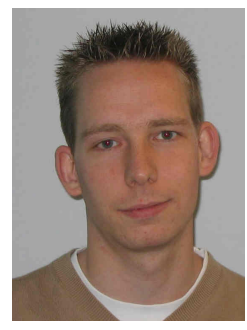

Vincent Bakker received his M.Sc. degree in Computer Science from the University of Twente in 2007 , with a minor certificate in Entrepreneurship. Currently he is working on his Ph.D. thesis researching domestic demand prediction for in home optimizations. Currently his interest are: machine learning, optimization modeling and large scale distributed (intelligent) systems.

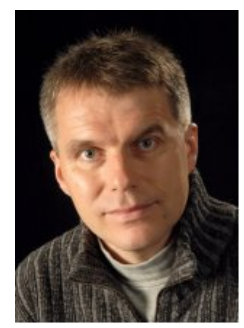

Johann L. Hurink received a Ph.D. degree at the University of Osnabrueck (Germany) in 1992 for a thesis on a scheduling problem occurring in the area of public transport. From 1992 until 1998 he has been an assistant professor at the same university working on local search methods and complex scheduling problems. From 1998 until 2005 he has been an assistant professor and from 2005 until 2009 an associated professor in the group Discrete Mathematics and Mathematical Programming at the department of Applied Mathematics at the University of Twente. Since 2009 he is a full professor of the same group.

Current work includes the application of optimization techniques and scheduling models to problems from logistics, health care, and telecommunication.

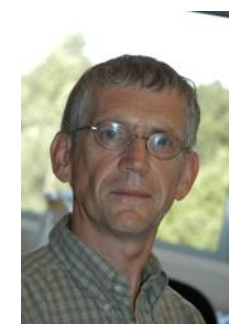

Gerard J.M. Smit received his M.Sc. degree in electrical engineering from the University of Twente. He then worked for four years in the research and development laboratory of Océ in Venlo. He finished his Ph.D. thesis entitled "the design of Central Switch communication systems for Multimedia Applications" in 1994. He has been a visiting researcher at the Computer Lab of the Cambridge University in 1994, and a visiting researcher at Lucent Technologies Bell Labs Innovations, New Jersey in 1998. Since 1999 he works in the Chameleon project, which investigates new hardware and software architectures for batterypowered hand-held computers. Currently his interests are: low-power communication, wireless multimedia communication, and reconfigurable architectures for energy reduction. Since 2006 he is full professor in the CAES chair (Computer Architectures for Embedded Systems) at the faculty EEMCS of the University of Twente. Prof. Smit has been and still is responsible of a number of research projects sponsored by the EC, industry and Dutch government in the field of multimedia and reconfigurable systems. 